\title{
SOME VARIETIES OF LIE RINGS
}

\author{
YIN CHEN AND RUNXUAN ZHANG
}

\begin{abstract}
Aвstract. In this paper, several theorems of Macdonald $[4,5]$ on the varieties of nilpotent groups will be generalized to the case of Lie rings. We consider three varieties of Lie rings of any characteristic associated with some equations (see Eqs. (1.1)-(1.3) below). We prove that each Lie ring in variety (1.1) is nilpotent of exponent at most $n+2$; if $L$ is a Lie ring in variety (1.2), then $L^{2}$ is nilpotent of exponent at most $n+1$; and each Lie ring in variety (1.3) is solvable of length at most $n+1$. Finally, we also discuss some varieties of solvable Lie rings and the varieties of Lie rings defined by the properties of subrings.
\end{abstract}

\section{INTRODUCTION}

A class of groups $\mathfrak{X}$ is a class whose members are groups and which has the following properties: (1) $\mathfrak{X}$ contains a group of order 1 ; (2) $H \simeq G \in \mathfrak{X}$ always implies that $H \in \mathfrak{X}$. A variety of groups is a class of groups which are defined by sets of equations. For example, all abelian groups form the variety associated with the equation $(x, y)=1$, where $(x, y)=x y x^{-1} y^{-1}$ is the commutator of elements $x$ and $y$. The study of varieties of groups is a subject with a long history. In 1961, Macdonald [4] proved that two kinds of varieties of groups associated with equations

$$
\begin{aligned}
\left(x, y_{1}, \cdots, y_{n}, x\right) & =1 \\
\left(x, y_{1}, \cdots, y_{n} ; x, y_{1}, \cdots, y_{m}\right) & =1
\end{aligned}
$$

are both nilpotent, and the corresponding nilpotent exponents was also calculated. In the same paper, Macdonald pointed out that the analogous results may be valid for some varieties of Lie rings, but he did not give any proof. Later, a lot of papers addressing the varieties of finite nilpotent groups and solvable groups appeared, such as $[1,2,3,5,6]$, in which the attention was focused on the subgroups of solvable groups with some restrictions.

As we know, there are a lot of similar concepts and results between finite group theory and finitedimensional Lie algebras. It is natural to bring some methods in finite group theory into the study of Lie algebras. Recently, Suanmali [8] (or [7]) used an analogous idea in the theory of group varieties to investigate the varieties of Lie algebras. She considered the exponent bound problem for some varieties of nilpotent Lie algebras, and extended Macdonald's results in $[4,5]$ to finite-dimensional Lie algebras over a field of characteristic not 2 and 3. Apart from this, very little results seems to be known about the varieties of Lie algebras. In this paper, we follow basically the routines of Macdonald and Suanmali, and reconsider the varieties of Lie rings. We also obtain some analogues of Macdonald and Suanmali's results. But, the advantage of our results is that the restriction of characteristic and the structure of vector spaces are taken away.

Date: August 28, 2018.

2010 Mathematics Subject Classification. 17A30;17B30.

Key words and phrases. Lie ring; nilpotency; variety. 
Specifically, our first purpose is to discuss the following three varieties of Lie rings of any characteristic associated with equations

$$
\begin{aligned}
\left(x, y_{1}, \cdots, y_{n}, x\right) & =0 ; \\
\left(x, y ; x_{1}, x_{2}, \cdots, x_{2 n-1}, x_{2 n} ; x, z\right) & =0 ; \\
\left(x, y ; x_{1}, x_{2}, \cdots, x_{2 n} ; x, z ; y_{1}, y_{2}, \cdots y_{m}\right) & =0 .
\end{aligned}
$$

We shall extend some results in [8], such as several corresponding Macdonald's theorems, to the varieties of Lie rings (Theorems 2.1, 2.3 and 2.4). The second purpose is to prove that the variety $V_{n}$ associated with the equation

$$
\left(x_{1}, \cdots, x_{n}, x_{n+1}, x_{n+2}\right)=\left(x_{1}, \cdots, x_{n}, x_{n+2}, x_{n+1}\right)
$$

is precisely the variety $V_{n}^{\prime}$ associated with the equation $\left(x_{1}, \cdots, x_{n} ; x_{n+1}, x_{n+2}\right)=0$; and the variety $P_{n}$ associated with the equation

$$
\left(x_{1}, \cdots, x_{i}, x_{i+1}, \cdots, x_{n}\right)=\left(x_{1}, \cdots, x_{i+1}, x_{i}, \cdots, x_{n}\right)
$$

is precisely the variety $P_{n}^{\prime}$ associated with the equation $\left(x_{1}, \cdots,\left(x_{i}, x_{i+1}\right), \cdots, x_{n}\right)=0$. As a corollary, we obtain that each Lie ring $L$ in the variety $P_{n}$ is solvable. We remark that the corresponding results in finite group theory are due to Levin ([2], Corollary 2.4) and Kikodze ([1], Corollary 2) respectively. Moreover, we shall show that a Lie ring $L$ satisfies $\left(x_{1}, x_{2}, \cdots, x_{n}\right)=\left(x_{1}, x_{\rho(2)}, \cdots, x_{\rho(n)}\right)$ for all nonidentical permutations $\rho$ of $\{2, \cdots, n\}$ if and only if $L$ is nilpotent of exponent at most $n-1$ (Theorem 2.8). In the last section, we also discuss some varieties of Lie ring defined by properties of subrings.

To do this, we first need some preliminaries.

A nonassociative ring $L$ with the multiplication $[-,-]$ is called a Lie ring if the following identities are satisfied:

$$
\begin{aligned}
{[x, y]+[y, x] } & =0 \text { (skew symmetry) } \\
{[x,[y, z]]+[y,[z, x]]+[z,[x, y]] } & =0 \text { (Jacobi identity). }
\end{aligned}
$$

for all $x, y, z \in L$. Let $x_{1}, \cdots, x_{n}, y_{1}, \cdots, y_{m}$ be elements in a Lie ring $L$, the commutator $\left(x_{1}, \cdots, x_{n}\right)$ in $L$ is defined as

$$
\left(x_{1}, x_{2}\right):=\left[x_{1}, x_{2}\right] \text {, and }\left(x_{1}, \cdots, x_{n}\right):=\left(\left(x_{1}, \cdots, x_{n-1}\right), x_{n}\right) \text {, for all } n>2 \text {. }
$$

Moreover, we define $\left(x_{1}, \cdots, x_{n} ; y_{1}, \cdots, y_{m}\right):=\left(\left(x_{1}, \cdots, x_{n}\right),\left(y_{1}, \cdots, y_{m}\right)\right)$.

Let $\rho$ be a permutation of $\{3, \cdots, k\}$. If a Lie ring $L$ satisfies the equation

$$
\left(x_{1}, x_{2}, x_{3}, \cdots, x_{k}\right)=\left(x_{1}, x_{2}, x_{\rho(3)}, \cdots, x_{\rho(k)}\right),
$$

we say that $L$ satisfies $C(k, \rho)$. If $L$ satisfies $C(k, \rho)$ for all permutations $\rho$ of $\{3, \cdots, k\}$, we say that $L$ satisfies $C(k)$.

For a Lie ring $L$, we define the lower central series

$$
L^{1}:=L \supset L^{2}:=[L, L] \supset \cdots \supset L^{n}:=\left[L, L^{n-1}\right] \supset \cdots
$$

for all $n=2,3, \cdots$. A Lie ring $L$ is said to be nilpotent if $L^{n+1}=\{0\}$ for some nonnegative integer $n$. If $L^{n+1}=\{0\}$ but $L^{n} \neq\{0\}$, then we say that $L$ has nilpotent exponent $n$. We also define a different kind of powers of $L$,

$$
L^{(0)}:=L \supset L^{(1)}:=[L, L] \supset \cdots \supset L^{(n+1)}:=\left[L^{(n)}, L^{(n)}\right] \supset \cdots .
$$


Every $L^{(n)}$ is an ideal of $L$ and $L$ is said to be solvable if $L^{(n+1)}=\{0\}$ for some nonnegative integer $n$. Moreover if $L^{(n+1)}=\{0\}$ but $L^{(n)} \neq\{0\}$, then we say that $L$ has solvable length $n$. A Lie ring $L$ is said to be abelian if $L^{2}=\{0\} ; L$ is said to be metabelian if $L^{2}$ is abelian.

A class of Lie rings $\mathfrak{Q}$ is a class whose members are Lie rings and which has the following properties: (1) $\mathfrak{Q}$ contains the zero ring; (2) $L \simeq E \in \mathfrak{L}$ always implies that $L \in \mathfrak{L}$. A variety of Lie rings is a class of Lie rings which are defined by sets of equations. For example, all abelian Lie rings form the variety associated with the equation $[x, y]=0$.

Lemma 1.1. Let $L$ be a Lie ring, then $L$ is metabelian if only and if $(u, v, x, y)=(u, v, y, x)$ for all elements $u, v, x, y$ in $L$.

Proof. If $L$ is metabelian, then $(u, v ; x, y)=0$. By Jacobi identity, we have

$$
[[u, v],[x, y]]+[x,[y,[u, v]]]+[y,[[u, v], x]]=0 .
$$

Thus $0=[x,[y,[u, v]]]+[y,[[u, v], x]]=(x ; u, v, y)+(u, v, x, y)=-(u, v, y, x)+(u, v, x, y)$. That is $(u, v, x, y)=(u, v, y, x)$. Conversely, we notice that $(u, v, x, y)=(u, v, y, x)$ implies that $[x,[y,[u, v]]]+$ $[y,[[u, v], x]]=0$. By Jacobi identity, we have $[u, v ; x, y]=0$, as desired.

Lemma 1.2. Let $L$ be a Lie ring of characteristic not 2. If $(x, y ; x, z)=0$ for all $x, y, z \in L$, then $L$ is metabelian.

Proof. Assume that $x=s+t$, then $0=(s+t, y ; s+t, z)=(s, y ; t, z)+(t, y ; s, z)$, and

$$
(s, y ; t, z)=-(t, y ; s, z) .
$$

Let $a, b, c, d$ be arbitrary elements in $L$, then $(a, b ; c, d)=-(c, d ; a, b)=(a, d ; c, b)=(d, a ; b, c)=$ $-(b, a ; d, c)=-(a, b ; c, d)$. Since the characteristic of $L$ is not $2,(a, b ; c, d)=0$. Thus $L$ is metabelian.

Lemma 1.3. Let $L$ be a Lie ring with $(x, y, x)=0$ for all $x, y \in L$.

(1) If the characteristic of $L$ is not 3 , then $L^{3}=0$.

(2) If the characteristic of $L$ is 3 , then $L$ is metabelian.

Proof. (1) Assume that $x, y, z$ are arbitrary elements in $L$, then

$$
0=(x+z, y, x+z)=(x, y, z)+(z, y, x) .
$$

Thus

$$
(x, y, z)=-(z, y, x)
$$

By Jacobi identity, we have

$$
\begin{aligned}
(x ; y, z) & =-(y ; z, x)-(z ; x, y)=(y, z, x)+(z, x, y) \\
& =-(x, z, y)-(x, z, y)=-2(x, z, y) \\
& =2(y, z, x)=-2(x ; y, z) .
\end{aligned}
$$

Notice that the characteristic of $L$ is not 3 , so $(x ; y, z)=0$ and $L^{3}=0$.

(2) Let $x, y, z$ are any elements in $L$, then $(x, z ; x, y)=(x, y, z, x)=-(x, z ; x, y)$. Since the characteristic of $L$ is $3,(x, z ; x, y)=0$. It follows from Lemma 1.2 that $L$ is metabelian.

Lemma 1.4. Let $S_{n}$ be the symmetric group of degree $n$ and $L$ be a Lie ring. Then the commutator of $\left(y_{1}, \cdots, y_{n}, x\right)$ can be expressed as the sum of $2^{n-1}$ commutators of the form $\pm\left(x, y_{\pi(1)}, \cdots, y_{\pi(n)}\right)$, where $\pi \in S_{n}$. 
Proof. Induction on $n$, see ([7], Lemma 3.1).

\section{Varieties of Nilpotent and Solvable Lie Rings}

In this section, we discuss the following three varieties of Lie ring associated with equations

$$
\begin{aligned}
\left(x, y_{1}, \cdots, y_{n}, x\right) & =0 ; \\
\left(x, y ; x_{1}, x_{2}, \cdots, x_{2 n-1}, x_{2 n} ; x, z\right) & =0 ; \\
\left(x, y ; x_{1}, x_{2}, \cdots, x_{2 n} ; x, z ; y_{1}, y_{2}, \cdots, y_{m}\right) & =0 .
\end{aligned}
$$

Theorem 2.1. Let L be a Lie ring with $\left(x, y_{1}, \cdots, y_{n}, x\right)=0$.

(1) If the characteristic of $L$ is not 3, then $L$ is nilpotent of exponent at most $n+1$.

(2) If the characteristic of $L$ is 3 , then $L$ is nilpotent of exponent at most $n+2$.

Proof. (1) The proof is same as that of Theorem 3.1 in [7].

(2) We prove this statement by induction on $n$. If the characteristic of $L$ is 3 and $n=1$, then $\left(x, y_{1}, x\right)=0$ implies that $L$ is metabelian by Lemma 1.3. Moreover, it follows from Lemma 1.1 that $(u, v, x, y)=$ $(u, v, y, x)$ for all elements $u, v, x, y$ in $L$. By (1.8),

$$
(x,(u, v), y)=(y,(u, v), x)=-(x,(u, v), y) .
$$

The characteristic of $L$ is not 2 , so $(u, v, x, y)=0$. This means that $L$ is nilpotent of exponent at most 3 . Now we suppose that $L$ is a Lie ring with $\left(x, y_{1}, \cdots, y_{n-1}, y_{n}, x\right)=0$. Let $x=u+v$ for some elements $u, v \in L$, then $0=\left(u+v, y_{1}, \cdots, y_{n}, u+v\right)=\left(u, y_{1}, \cdots, y_{n}, v\right)+\left(v, y_{1}, \cdots, y_{n}, u\right)$. It follows from Lemma 1.4 that

$$
\begin{aligned}
\left(u, y_{1}, \cdots, y_{n}, v\right) & =-\left(v, y_{1}, \cdots, y_{n}, u\right) \\
& = \pm\left[\sum_{j=1}^{2^{n-1}}\left(y_{n}, y_{1_{j}}, \cdots, y_{n_{j}},\right), u\right] \\
& = \pm \sum_{j=1}^{2^{n-1}}\left(y_{n}, y_{1_{j}}, \cdots, y_{n_{j}}, u\right),
\end{aligned}
$$

where $y_{1_{j}}, \cdots, y_{n_{j}}$, are some permutations of $v, y_{1}, \cdots, y_{n-1}$ respectively. Thus

$$
\left(u, y_{1}, \cdots, y_{n-1}, u, v\right)= \pm \sum_{j=1}^{2^{n-1}}\left(u, y_{1_{j}}, \cdots, y_{n_{j}}, u\right)=0 .
$$

Namely, $\left(u, y_{1}, \cdots, y_{n-1}, u\right)$ belongs to $C(L)$, the center of $L$. So the factor ring $L / C(L)$ satisfies the induction hypothesis, and it is nilpotent of exponent at most $n+1$. Thus for all $x, y_{1}, \cdots, y_{n}, y$ in $L / C(L)$, we have $\left(x, y_{1}, \cdots, y_{n}, y\right) \in C(L)$ and $\left(x, y_{1}, \cdots, y_{n}, y, z\right)=0$ for each $z \in L$. Therefore $L$ is nilpotent of exponent at most $n+2$.

Lemma 2.2. Let $S_{2 n}$ be the symmetric group of degree $2 n, n \geq 1$ and $L$ a Lie ring. Then the commutator of $\left(x_{1}, x_{2} ; x_{3}, x_{4} ; \cdots ; x_{2 n-1}, x_{2 n}\right)$ can be expressed as the sum of $2^{n-1}$ commutators of the form $\pm\left(x_{\pi(1)}, x_{\pi(2)}, \cdots, x_{\pi(2 n)}\right)$, where $\pi \in S_{2 n}$.

Proof. We prove this result by induction on $n$. When $n=2$, the Jacobi identity implies that

$$
\left(x_{1}, x_{2} ; x_{3}, x_{4}\right)=\left(x_{1}, x_{2}, x_{3}, x_{4}\right)-\left(x_{1}, x_{2}, x_{4}, x_{3}\right) \text {. }
$$


The result holds. When $n \geq 2$, we let $z=\left(x_{1}, x_{2} ; x_{3}, x_{4} ; \cdots ; x_{2 n-3}, x_{2 n-2}\right)$. Then

$$
\begin{aligned}
{\left[z,\left[x_{2 n-1}, x_{2 n}\right]\right] } & =-\left[x_{2 n-1},\left[x_{2 n}, z\right]\right]-\left[x_{2 n},\left[z, x_{2 n-1}\right]\right] \\
& =\left(z, x_{2 n-1}, x_{2 n}\right)-\left(z, x_{2 n}, x_{2 n-1}\right) .
\end{aligned}
$$

By induction hypothesis, we assume that

$$
z= \pm \sum_{j=1}^{2^{n-2}}\left(x_{1}, x_{2}, x_{\pi_{j}(1)}, x_{\pi_{j}(2)}, \cdots, x_{\pi_{j}(2 n-2)}\right) .
$$

Then

$$
\begin{aligned}
& \left(z, x_{2 n-1}, x_{2 n}\right)= \pm \sum_{j=1}^{2^{n-2}}\left(x_{1}, x_{2}, x_{\pi_{j}(1)}, x_{\pi_{j}(2)}, \cdots, x_{\pi_{j}(2 n-2)}, x_{2 n-1}, x_{2 n}\right) \\
& \left(z, x_{2 n}, x_{2 n-1}\right)= \pm \sum_{j=1}^{2^{n-2}}\left(x_{1}, x_{2}, x_{\pi_{j}(1)}, x_{\pi_{j}(2)}, \cdots, x_{\pi_{j}(2 n-2)}, x_{2 n}, x_{2 n-1}\right) .
\end{aligned}
$$

Thus

$$
\left(z ; x_{2 n-1}, x_{2 n}\right)= \pm \sum_{j=1}^{2^{n-1}}\left(x_{1}, x_{2}, x_{\pi_{j}(1)}, x_{\pi_{j}(2)}, \cdots, x_{\pi_{j}(2 n)}\right)
$$

as desired.

Theorem 2.3. Let $L$ be a Lie ring with $\left(x, y ; x_{1}, x_{2}, \cdots, x_{2 n-1}, x_{2 n} ; x, z\right)=0$. Then $L^{2}$ is nilpotent of exponent at most $n+1$.

Proof. Let $u=(s, y), x_{i}^{\prime}=\left(x_{2 i-1}, x_{2 i}\right)$ and $v=(t, z)$. It follows from Lemma 1.4 that

$$
\begin{aligned}
\left(s, y ; x_{1}, x_{2} ; x_{3}, x_{4} ; \cdots ; x_{2 n-1}, x_{2 n} ; t, z\right) & =\left(u, x_{1}^{\prime}, x_{2}^{\prime}, \cdots, x_{n}^{\prime}, v\right) \\
& = \pm\left[\sum_{j=1}^{2^{n-1}}\left(x_{n}^{\prime}, x_{1_{j}}^{\prime}, \cdots, x_{n_{j}}^{\prime}\right), v\right] \\
& = \pm \sum_{j=1}^{2^{n-1}}\left(x_{n}^{\prime}, x_{1_{j}}^{\prime}, \cdots, x_{n_{j}}^{\prime}, v\right),
\end{aligned}
$$

where $x_{1_{j}}^{\prime}, \cdots, x_{n_{j}}^{\prime}$, are some permutations of $u, x_{1}^{\prime}, \cdots, x_{n-1}^{\prime}$ respectively. Let $x_{n}^{\prime}=v=(t, z)$. By Lemma 2.2, we obtain

$$
\begin{aligned}
\left(s, y ; x_{1}, x_{2} ; x_{3}, x_{4} ; \cdots ; x_{2 n-1}, x_{2 n} ; t, z\right) & = \pm \sum_{j=1}^{2^{n-1}}\left(t, z ; x_{1_{j}}^{\prime}, \cdots, x_{n_{j}}^{\prime} ; t, z\right) \\
& = \pm \sum_{j=1}^{2^{n-1}}\left(t, z ; x_{1_{j}}, x_{2_{j}}, \cdots, x_{n_{j}} ; t, z\right)=0 .
\end{aligned}
$$

Hence $L^{2}$ is nilpotent of exponent at most $n+1$.

Theorem 2.4. Let L be a Lie ring with $\left(x, y ; x_{1}, x_{2}, \cdots, x_{2 n} ; x, z ; y_{1}, y_{2}, \cdots, y_{m}\right)=0$, where $2 n \geq m \geq 1$ and $n \geq 1$. Then $L$ is solvable of length at most $n+1$. 
Proof. We write $C_{L}\left(L^{m}\right)$ for the centralizer of $L^{m}$ in $L$. The factor ring $L / C_{L}\left(L^{m}\right)$ satisfies the identity $\left(x, y ; x_{1}, x_{2}, \cdots, x_{2 n} ; x, z\right)=0$, it follows from Theorem 2.3 that $\left(L / C_{L}\left(L^{m}\right)\right)^{2}$ is nilpotent of exponent at most $n+1$. Thus

$$
\left(s, y ; x_{1}, x_{2} ; x_{3}, x_{4} ; \cdots ; x_{2 n-1}, x_{2 n} ; t, z\right) \in C_{L}\left(L^{m}\right)
$$

and $\left(s, y ; x_{1}, x_{2} ; x_{3}, x_{4} ; \cdots ; x_{2 n-1}, x_{2 n} ; t, z ; y_{1}, y_{2}, \cdots, y_{m}\right)=0$. From Lemma 2.2 , we know that each element $u$ in $L^{(n+2)}$ can be expressed as a finite sum of the form

$$
\pm\left(x_{\pi(1)}, x_{\pi(2)} ; x_{\pi(3)}, x_{\pi(4)} ; \cdots ; x_{\pi\left(2^{n+2}-1\right)}, x_{\pi\left(2^{n+2}\right)}\right),
$$

where $\pi \in S_{2^{n+2}}$. That is

$$
u= \pm \sum\left(x_{\pi(1)}, x_{\pi(2)} ; \cdots ; x_{\pi(2 n+3)}, x_{\pi(2 n+4)} ; x_{\sigma(2 n+5)}, x_{\sigma(2 n+6)}, \cdots, x_{\sigma\left(2^{n+2}\right)}\right),
$$

for some $\pi, \sigma \in S_{2^{n+2}}$. We note that $2^{n+2}-(2 n+4) \geq m$. Hence $L^{(n+2)}=\{0\}$, as desired.

Proposition 2.5. The variety $V_{n}$ associated with the equation

$$
\left(x_{1}, \cdots, x_{n}, x_{n+1}, x_{n+2}\right)=\left(x_{1}, \cdots, x_{n}, x_{n+2}, x_{n+1}\right)
$$

is precisely the variety $V_{n}^{\prime}$ associated with the equation $\left(x_{1}, \cdots, x_{n} ; x_{n+1}, x_{n+2}\right)=0$. In particular, $V_{2}$ is the variety of metabelian Lie rings.

Proof. Let $z=\left(x_{1}, \cdots, x_{n}\right)$. This statement follows immediately from Jacobi identity.

Theorem 2.6. The variety $P_{n}$ associated with the equation

$$
\left(x_{1}, \cdots, x_{i}, x_{i+1}, \cdots, x_{n}\right)=\left(x_{1}, \cdots, x_{i+1}, x_{i}, \cdots, x_{n}\right)
$$

is precisely the variety $P_{n}^{\prime}$ associated with the equation $\left(x_{1}, \cdots,\left(x_{i}, x_{i+1}\right), \cdots, x_{n}\right)=0$. Moreover, each Lie ring $L$ in $P_{n}$ is solvable.

Proof. Let $a=\left(x_{1}, \cdots, x_{i-1}\right), b=\left(x_{i+2}, \cdots, x_{n}\right)$. It follows from Jacobi identity that

$$
\begin{aligned}
0 & =\left(\left(a,\left(x_{i}, x_{i+1}\right)\right)+\left(x_{i},\left(x_{i+1}, a\right)\right)+\left(x_{i+1},\left(a, x_{i}\right)\right), b\right) \\
& =\left(\left(a,\left(x_{i}, x_{i+1}\right)\right)+\left(\left(a, x_{i+1}\right), x_{i}\right)-\left(\left(a, x_{i}\right), x_{i+1}\right), b\right) \\
& =\left(a,\left(x_{i}, x_{i+1}\right), b\right)+\left(a, x_{i+1}, x_{i}, b\right)-\left(a, x_{i}, x_{i+1}, b\right) .
\end{aligned}
$$

Thus the equation $\left(x_{1}, \cdots, x_{i}, x_{i+1}, \cdots, x_{n}\right)=\left(x_{1}, \cdots, x_{i+1}, x_{i}, \cdots, x_{n}\right)$ is equivalent to

$$
\left(x_{1}, \cdots,\left(x_{i}, x_{i+1}\right), \cdots, x_{n}\right)=0 .
$$

It is easy to see that $P_{2}$ is the variety of abelian Lie rings. We use induction on $n$ to prove the solvability of a Lie ring $L$ in $P_{n}$. Since $\left(x_{1}, \cdots,\left(x_{i}, x_{i+1}\right), \cdots, x_{n}\right)=0$,

$$
\left(x_{1}, \cdots,\left(x_{i}, x_{i+1}\right), \cdots, x_{n-1}\right)(i<n-1)
$$

belongs to $C(L)$, the center of $L$. That is $\left(x_{1}, \cdots,\left(x_{i}, x_{i+1}\right), \cdots, x_{n-1}\right)=0$ in $L / C(L)$. By induction hypothesis, we conclude that the factor ring $L / C(L)$ is solvable, and thus $L$ is also solvable. If $\left(x_{1}, \cdots, x_{n-2},\left(x_{n-1}, x_{n}\right)\right)=$ 0 , we observe that the equations $\left(x_{n-1}, x_{n} ; x_{1}, \cdots, x_{n-2}\right)=0$ and $\left(x_{1}, x_{2} ; x_{3}, \cdots, x_{n}\right)=0$ are equivalent. Hence $L$ is also solvable.

Lemma 2.7. Let $L$ be a Lie ring with $\left(x_{1}, x_{2}, \cdots, x_{n} ; y_{1}, y_{2}\right)=0$, where $n \geq 2$. If $L / C(L)$ satisfies $C(n+1)$, then L satisfies $C(n+2)$. 
Proof. By Jacobi identity, we see that

$$
[a,[x, y]]=0 \Leftrightarrow(a, x, y)=(a, y, x) .
$$

Thus $L$ satisfies $C\left(n+2, \rho_{1}\right)$ for $\rho_{1}=(n+1 n+2)$. Since $L / C(L)$ satisfies $C(n+1), L / C(L)$ satisfies $C\left(n+1, \rho_{2}\right)$ for $\rho_{2}=(34 \cdots n+1)$. Namely,

$$
\left(\left(x_{1}, x_{2}, x_{3}, \cdots, x_{n+1}\right)-\left(x_{1}, x_{2}, x_{4}, \cdots, x_{n+1}, x_{3}\right), x_{n+2}\right)=0 .
$$

Thus $L$ satisfies $C\left(n+2, \rho_{2}\right)$. Notice that $L$ satisfies $C\left(n+2, \rho_{1}\right)$ and $\rho_{1}, \rho_{2}$ generate the group of all permutations of $\{3,4 \cdots, n+2\}$, thus $L$ satisfies $C(n+2)$.

Theorem 2.8. Let L be a Lie ring, then L satisfies

$$
\left(x_{1}, x_{2}, \cdots, x_{n}\right)=\left(x_{1}, x_{\rho(2)}, \cdots, x_{\rho(n)}\right)
$$

for all nonidentical permutations $\rho$ of $\{2, \cdots, n\}$, if and only if $L$ is nilpotent of exponent at most $n-1$.

Proof. We shall use the induction on $n$. For $n=3,\left(x_{1}, x_{2}, x_{3}\right)=\left(x_{1}, x_{3}, x_{2}\right)$ if and only if $\left[x_{1},\left[x_{2}, x_{3}\right]\right]=$ 0 by (2.2). Assume that $L$ satisfies the hypothesis for $n>3$. By (2.2), we deduce that $L$ satisfies $\left(x_{1}, x_{2}, \cdots ; x_{n-1}, x_{n}\right)=0$. Notice that $L$ satisfies (2.3) for all nonidentical permutations $\rho$. A similar argument as in the proof of Lemma 2.7 implies that $L / C(L)$ satisfies (2.3) with $n$ replaced by $n-1$ for any permutation $\sigma$ of $\{2, \cdots, n-1\}$. Induction hypothesis yields that $L / C(L)$ is nilpotent of exponent at most $n-2$, and $L$ is nilpotent of exponent at most $n-1$. The proof of the converse is trivial.

\section{Varieties Defined by Properties of Subrings}

In this section, we return to some of Macdonald's ideas in [5] which were originally used to discuss the group-theoretic problems.

Theorem 3.1. Let L be a Lie ring.

(1) If $L$ has characteristic not 3, then every n-generated subring of $L$ is nilpotent of exponent at most $n$ if and only if $L$ is also nilpotent of exponent at most $n$.

(2) If $L$ has characteristic 3, then every $n$-generated subring of $L$ is nilpotent of exponent at most $n$ if and only if $L$ is also nilpotent of exponent at most $n+1$.

Proof. $(\Rightarrow)$ Let $x, y, x_{1}, \cdots, x_{n-2}$ be any elements of $L$. The subring $\left\langle x, y, x_{1}, \cdots, x_{n-2}\right\rangle$ is nilpotent of exponent at most $n$. Let $a=\left(x_{1}, \cdots, x_{n-2}\right)$, then

$$
\begin{aligned}
(x, y, x, a) & =-(a,(x, y), x)-(x, a ; x, y) \\
& =(a, x ; x, y)-(a,(x, y), x) \\
& =(a, x, x, y)-(a, x, y, x)-((-(x ; y, a)-(y ; a, x)), x) \\
& =(a, x, x, y)-(a, x, y, x)+(a, y, x, x)-(a, x, y, x)=0 .
\end{aligned}
$$

Namely $\left(x, y, x ; x_{1}, \cdots, x_{n-2}\right)=0$, and thus $(x, y, x)$ in $C_{L}\left(L^{n-2}\right)$, the centralizer of $L^{n-2}$ in $L$. Notice that $L / C_{L}\left(L^{n-2}\right)$ satisfies the conditions in Lemma 1.3. It follows that $L / C_{L}\left(L^{n-2}\right)$ has exponent no more than 3 in case of characteristic not 3 , and is metabelian in case of characteristic 3 . Therefore $L$ is nilpotent of exponent at most $n(\operatorname{char} L \neq 3)$ and $n+1(\operatorname{char} L=3)$.

$(\Leftarrow)$ Obviously.

This results with Theorem 2.1 give the following conclusions.

Corollary 3.2. Let $\mathfrak{Q}$ be the variety of Lie rings. 
(1) If each Lie ring in $\mathfrak{Q}$ is of characteristic not 3 and with the property that every $n$-generated subring is nilpotent of exponent at most $n$, then $\mathfrak{Q}$ is determined by the equation

$$
\left(x, x_{1}, \cdots, x_{n-1}, x\right)=0 .
$$

(2) If each Lie ring in $\mathfrak{Q}$ is of characteristic 3 and with the property that every $n$-generated subring is nilpotent of exponent at most $n+1$, then $\mathfrak{Q}$ is determined by the equation

$$
\left(x, x_{1}, \cdots, x_{n-1}, x\right)=0 .
$$

With an analogous argument, we have

Theorem 3.3. The variety of Lie rings of characteristic not 2 in which every subring with $2^{n+1}-1$ generators is solvable of length at most $n$, is determined by the equation

$$
\left(x, y ; x_{1}, x_{2}, \cdots, x_{2 n-2} ; x, z ; y_{1}, y_{2}, \cdots, y_{2 n-2}\right)=0 \text {, }
$$

where $n \geq 2$.

Proof. By Theorem 2.4, it suffices to show that a Lie ring $L$ with every subring with $2^{n+1}-1$ generators is solvable of length at most $n$, is solvable of length at most $n$. If $n=2$, then for all $x, y, x_{1}, x_{2}, x, z, y_{1}, y_{2}$ in $L,\left(\left(x, y ; x_{1}, x_{2}\right),\left(x, z ; y_{1}, y_{2}\right)\right)=0$. Let $z=y$, then

$$
\left(\left(x, y ; x_{1}, x_{2}\right),\left(x, y ; y_{1}, y_{2}\right)\right)=0 .
$$

That means that $L^{(1)}$ satisfies the condition of Lemma 1.2 , so $L^{(1)}$ is metabelian. Thus $L^{(3)}=0$ and $L$ is solvable of length at most 2 .

Assume that $x, y, x_{1}, x_{2}, \cdots, x_{2^{n}-2}, x, z, y_{1}, y_{2}, \cdots, y_{2^{n}-2}$ are $2^{n+1}-1$ arbitrary elements of $L$. Since the subring $\left\langle x, y, x_{1}, x_{2}, \cdots, x_{2^{n}-2}, x, z, y_{1}, y_{2}, \cdots, y_{2^{n}-2}\right\rangle$ is solvable of length at most $n$,

$$
\left.\left.\left.\left[\cdots\left[[x, y],\left[x_{1}, x_{2}\right]\right] \cdots\right], \cdots, x_{2^{n}-2}\right] ;\left[\cdots\left[[x, z],\left[y_{1}, y_{2}\right]\right] \cdots\right], \cdots, y_{2^{n}-2}\right] \cdots\right]=0 .
$$

We let $z=y$, then

$$
\left.\left.\left.\left[\cdots\left[[x, y],\left[x_{1}, x_{2}\right]\right] \cdots\right], \cdots, x_{2^{n}-2}\right] ;\left[\cdots\left[[x, y],\left[y_{1}, y_{2}\right]\right] \cdots\right], \cdots, y_{2^{n}-2}\right] \cdots\right]=0 .
$$

That is, in $L^{(1)}$, every subring with $2^{n}-1$ generators is solvable of length at most $n-1$. By induction hypothesis, it follows that $L^{(1)}$ is solvable of length at most $n-1$. Thus $L^{(n+1)}=0$, as desired.

\section{Acknowledgments}

The authors are grateful to an anonymous referee for his (or her) valuable comments and suggestions on the first version of the article. This work was supported by the Fundamental Research Funds for the Central Universities (No. 111494343) and NSF of China (No.11026136).

\section{REFERENCES}

[1] É. Kikodze, On certain identities in groups. Izv. Akad. Nauk SSSR Ser. Mat. 31 (1967) 269-274.

[2] F. Levin, On some varieties of soluble groups I. Math. Z. 85 (1964) 369-372.

[3] F. Levin, On some varieties of soluble groups II. Math. Z.103 (1968) 162-172.

[4] I. Macdonald, On certain varieties of groups. Math. Z. 76 (1961) 270-282.

[5] I. Macdonald, On certain varieties of groups II. Math. Z. 78 (1962) 175-188.

[6] I. Macdonald, Some metabelian-like varieties of groups. Amer. Math. Monthly 72 (1965) 159-162.

[7] S. Suanmali, On the relationship between the class of a Lie algebra and the classes of its subalgebras. PhD thesis, North Carolina State University (2007).

[8] S. Suanmali, On the relationship between the class of a Lie algebra and the classes of its subalgebras. Internat. J. Algebra Comput. 18 (2008) 83-95. 
School of Mathematics and Statistics, Northeast Normal University, Changchun 130024, P.R. China and Chern Institute of Mathematics, Nankai University, Tianjin 300071, P.R. China

E-mail address: ychen@nenu.edu.cn

School of Mathematics and Statistics, Northeast Normal University, Changchun 130024, P.R. China

E-mail address: zhangrx728@nenu. edu.cn 